\title{
A GEOMETRIC THEORY OF SOLUTION OF LINEAR INEQUALITIES*
}

\author{
BY \\ RUTH WYCKLIFFE STOKES
}

1. Introduction. The general theory of solution of linear inequalities has been treated previously by at least two authors, and particular aspects of the subject have been discussed by others.

Minkowski's $\dagger$ treatment is built around the theorem that the general solution of a set of linear homogeneous inequalities of the form

$$
\sum_{i=1}^{n} \lambda^{i} x_{i}^{i} \geqq 0 \quad(j=1, \cdots, N),
$$

in which the $\lambda$ 's are the unknowns, is a linear homogeneous combination of a fundamental set of particular solutions. Because of this, his theory has a marked analogy with the theory of linear equations. His treatment of (1.1) is theoretically complete, and his ideas are of such a fundamental nature that they will necessarily appear in some guise in any thorough discussion of the subject.

Dines $\ddagger$ gives a necessary and sufficient condition that a system of the form

$$
\sum_{i=1}^{n} \lambda^{i} x_{i}^{i}>0
$$

have a solution, and a criterion for the degree of arbitrariness of the general solution. These are stated in terms of what he calls the $I$-rank of the system, this quantity being defined in terms of a sequence of matrices formed from

* Presented to the Society, April 3, 1931; received by the editors in March, 1931; presented, in substance, as a Doctor's dissertation at Duke University.

$\dagger$ H. Minkowski, Geometrie der Zahlen, Leipzig, 1910, pp. 40-45.

$\ddagger$ L. L. Dines, Systems of linear inequalities, Annals of Mathematics, (2), vol. 20 (1918-19), pp. 191-199.

Other articles by this author on related subjects are:

Definite linear dependence, Annals of Mathematics, (2), vol. 27 (1926), pp. 57-64.

Note on certain associated systems of linear equalities and inequalities, Annals of Mathematics, (2), vol. 28 (1926-27), pp. 41-42.

On positive solutions of a system of linear equations, Annals of Mathematics, (2), vol. 28 (1926-27), pp. 386-392.

Linear inequalities and some related properties of functions, Bulletin of the American Mathematical Society, vol. 36 (1930), pp. 393-405. 
the matrix of the coefficients. It is in this particular that his theory has its analogy (not so close as Minkowski's) with the theory of linear homogeneous equations. In addition, he gives a method of finding the general solution of (1.2), which appears in unattractive form due to its lack of symmetry. ${ }^{*} \mathrm{He}$ has also considered particular problems of related types such as the determination of a particular positive solution of a system of linear homogeneous equations.

Minkowski also considers briefly system (1.2). He gives a necessary and sufficient condition that it have a solution but does not give its general solution.

Carver $\dagger$ gives a necessary and sufficient condition for the non-existence of solutions of (1.2). He also considers questions of independence and equivalence.

The present paper is designed to obtain results combining the advantages of the work just cited, and extending it. The theory of systems (1.1) is developed in such a form that it readily gives a solution of any system formed by uniting a system (1.1), a system (1.2), and a system

$$
\sum_{i=1}^{n} \lambda^{i} x_{i}^{j}=0
$$

We thus have a theory which has three extreme cases: Minkowski's system, Dines' system, and a system of linear homogeneous equations. The present paper exhibits the general solution of (1.1) in Minkowski's form and the general solution of the combined problem in a similar form. It makes the numerical work of solution center about a single matrix formed from the matrix of coefficients.

The method of developing the necessary theory is geometric in the following sense. For three dimensions the theorems are capable of visualization and in fact are almost intuitively obvious. When proofs of them are formulated in terms of analytic geometry, their extension to $n$ dimensions, together with the necessary proofs, is immediate. Although Minkowski's treatment occurs in a book on the geometry of numbers and occasionally employs geometric nomenclature, both his treatment and Dines' are essentially analytic.

The two- and three-dimensional cases are treated in detail at the start in order to exhibit the geometric character of the reasoning ( $\$ \$ 4$ and 5).

A geometric method distinct from that of the present paper is employed

* L. L. Dines, Systems of linear inequalities, Annals of Mathematics, (2), vol. 20 (1918-19), p. 199.

$\dagger$ W. B. Carver, Systems of linear inequalities, Annals of Mathematics, (2), vol. 23 (1921), pp. 212-220. 
by Lovitt* in the solution of a particular problem in three unknowns. $\dagger$

2. Statement of the problem. It is proposed to solve the system of inequalities (1.1) in which the coefficients $x$ are known and the $\lambda$ 's are to be determined.

Every system (1.1) is satisfied by $\lambda^{i}=0$. This will accordingly be called the trivial solution. Any other solution will be by definition non-trivial.

In order to apply geometric methods to the solution of this problem, it is convenient to recall certain properties of euclidean space of $n$ dimensions referred to a rectangular cartesian coördinate system, or to a system arising from a rectangular cartesian system by a linear homogeneous transformation. $\ddagger$

A linear homogeneous expression in the coördinates $x$, at least one of whose coefficients is not zero, defines an oriented $(n-1)$-flat (passing through the origin§). Points whose coördinates make this expression positive lie on the positive side of the $(n-1)$-flat, those making it negative lie on the negative side, and those making it zero lie on it.

Two oriented $(n-1)$-flats are the same if and only if the expression defining one is a positive multiple of that defining the other.

The totality of points on an oriented $(n-1)$-flat forms an unoriented $(n-1)$-flat whose equation is obtained by equating to zero the expression defining the oriented $(n-1)$-flat.

An $(n-1)$-flat (oriented or unoriented) separates a pair of points if and only if the substitution of the coördinates of those points in the expression defining it gives opposite signs.

To each condition (1.1) there corresponds a point $\left(x_{1}{ }^{j}, \cdots, x_{n}{ }^{j}\right)$ in $n$ space. The given system is thus represented geometrically by a set of points which we shall denote by $S_{n}$.

* W. V. Lovitt, Preferential voting, American Mathematical Monthly, vol. 23 (1916), pp. 363-66.

$\dagger$ In order to render the bibliography more nearly complete, we cite here two papers whose existence has been called to our attention, but which we have not seen. They are by M. Fujiwara and appear in the 1928 and 1930 volumes of the Proceedings of the Imperial Academy, Tokyo.

$\ddagger$ We find it desirable to describe the admissible coördinate systems in this manner. Any given set of data is most conveniently represented by interpreting the coördinates as rectangular cartesian. But this interpretation of $x, y, z$ being once chosen (we take $n=3$ for illustration), it is convenient at times to choose $x, y$ as the coördinates in a plane through the origin oblique to the coördinate planes, and $x, y$ are not rectangular cartesian coördinates for that plane.

\& For our purpose it suffices to consider only flats containing the origin, and we shall understand, even in the absence of express mention of the fact, that every geometric element of one or more dimensions contains the origin, except in $\$ 15$.

I Dines, Annals of Mathematics, (2), vol. 27 (1926), pp. 58-59, mentions that the representation of each condition can be accomplished by means of a point or by means of a vector. He uses the latter representation to describe the condition for non-existence of a solution of (1.2). He does not represent the solution geometrically, however, and does not employ a geometric method of reasoning in obtain- 
To any non-trivial solution $\lambda$ of (1.1) there corresponds an oriented $(n-1)$-flat determined by the expression

$$
\sum_{i=1}^{n} \lambda^{i} x_{i}
$$

such that none of the points of $S_{n}$ is on the negative side of it.*

Conversely, the coefficients of any oriented $(n-1)$-flat having this property give a non-trivial solution of (1.1).

Two solutions of (1.1) will be called the same if the corresponding two $(n-1)$-flats are the same. Hence the solution of (1.1) can be accomplished by finding every oriented $(n-1)$-flat having the property that no point of $S_{n}$ lies on the negative side of it.

A necessary condition on a solution is that the corresponding unoriented $(n-1)$-flat do not separate any two points of the set.

3. Reduction of dimensionality. If the rank of the matrix

$$
\left\|x_{i}^{j}\right\|
$$

is $r<n$, the given points $S_{n}$ lie in an $r$-flat, $\Pi_{r}$. As coördinates in that $r$-flat we may take any $r$ of the coördinates $x$ such that the matrix formed from the corresponding columns of (3.1) is of rank $r$. For convenience of language we assume that $x_{1}, \cdots, x_{r}$ are the coördinates in $\Pi_{r}$. The remaining $n-r$ coordinates $x_{r+1}, \cdots, x_{n}$ are linear homogeneous functions of them.

If an unoriented $(n-1)$-flat contains $\Pi_{r}$, either oriented $(n-1)$-flat associated with it gives a solution. This solution, being of the particular type which makes every left member of (1.1) zero, will be called an equality solution.

If an oriented ( $n-1)$-flat defined by an expression

$$
\sigma(x)
$$

does not contain $\Pi_{r}$, then it meets $\Pi_{r}$ in an oriented $(r-1)$-flat whose defining expression, when it is regarded as an oriented hyperplane in the space of $r$ dimensions, can be obtained by eliminating $x_{r+1}, \cdots, x_{n}$ from (3.2) by means of the equations defining $\Pi_{r}$. Hence (3.2) regarded as an $(n-1)$-flat in $n$ space separates two points of $S_{n}$, if and only if it separates them when it is

ing his results. Actually, when the coördinates are rectangular cartesian, the vector used by Dines in describing the condition for the non-existence of solutions of (1.2) is the directed normal to the $(n-1)$-flat which we employ to represent the solution.

* Following a well established practice in the application of geometric methods to analysis, we regard the solution, the corresponding $(n-1)$-flat, and its defining linear homogeneous expression as identical. 
regarded as an $(r-1)$-flat in $\Pi_{r}$. Given an $(r-1)$-flat defining a solution in $\Pi_{r}$, any $(n-1)$-flat containing it, when given the proper orientation, defines a solution in $n$-dimensional space, and, conversely, every solution not containing $\Pi_{r}$ in $n$-dimensional space is obtained in this manner.

Let $\sigma$ be the general solution of the problem in $\Pi_{r}$. Let the expressions whose vanishing defines $\Pi_{r}$ be $u_{r+1}, \cdots, u_{n}$. Then

$$
\sigma+\sum_{i=r+1}^{n} a^{i} u_{i}
$$

where the $a$ 's are arbitrary real constants, is an (n-1)-flat whose trace on $\Pi_{r}$ is $\sigma$. Hence (3.3) is a solution in $n$ dimensions. Conversely, any solution in $n$ dimensions is a linear homogeneous function of the $x$ 's which reduces to $\sigma$ on $\Pi_{r}$ and has, therefore, the form (3.3). Accordingly, (3.3) gives the general solution in $n$ dimensions. The solutions containing $\Pi_{r}$ are obtained by making $\sigma=0$ in (3.3).

Because of the above result, in the sequel we need only indicate how to solve (1.1) when its matrix is of rank $n$, i.e., when the set $S_{n}$ (plus the origin) is actually $n$-dimensional.

A useful consequence of the above is

THEOREM 1. The intersection of two solutions $\sigma, \sigma^{\prime}$ is an $(n-2)$-flat which is a solution of the problem for $n-1$ dimensions defined by the points of the set in $\sigma$, and also of the problem defined by the points of the set in $\sigma^{\prime}$.

Since $\sigma$ is a solution for the whole set of points, it is a solution for the points contained in $\sigma^{\prime}$ and by the result given above must intersect $\sigma^{\prime}$ in a solution for the set of points in $\sigma^{\prime}$.

4. Linear homogeneous inequalities in two variables. In accordance with the results developed in the last section, it is sufficient to discuss the case where not all the representative points lie on a single line through the origin.

A non-trivial solution is necessarily of one of two types: it may contain a point of the set other than the origin, in which case it will be called a fundamental solution; or it may contain no such point.

Suppose the system proposed has a solution $\sigma$ of the latter type. If the line representing it be rotated without passing through a point of the set, it continues to represent a solution. If it be so rotated until it contains a point of the set, it becomes a fundamental solution. As the rotation can take place in either sense, two fundamental solutions are thus obtained. If these fundamental solutions coincided, the set of points would be collinear with the 
origin, contrary to hypothesis. Hence there exist two fundamental solutions $\sigma_{1}$ and $\sigma_{2}$. Moreover, we can write

$$
\sigma=a \sigma_{1}+b \sigma_{2}
$$

where $a$ and $b$ are constants. Since $\sigma$ and $\sigma_{1}$ are positive for any point of the set on $\sigma_{2}, a$ must be positive. In similar fashion $b$ is proved positive.

Conversely, if the constants in the right member of (4.1) are given arbitrary positive values, the expression $\sigma$ is positive for any point of the set because $\sigma_{1}$ and $\sigma_{2}$ have non-negative values for any point of the set and vanish simultaneously at no point of the set.

THEOREM 2. The system

$$
\lambda x^{j}+\mu y^{j} \geqq 0 \quad(j=1,2, \cdots, N),
$$

whose matrix is of rank two, has a solution which is not a fundamental solution if and only if it has two fundamental solutions $\sigma_{1}$ and $\sigma_{2}$. The general solution of (4.2) which contains no point of the set is then expressible as

$$
a \sigma_{1}+b \sigma_{2}
$$

where $a$ and $b$ are arbitrary positive constants.

A consequence of the above is that if (4.2) has only one fundamental solution, that fundamental solution is the only solution.

THEOREM 3. The system

$$
\lambda x^{j}+\mu y^{j} \geqq 0 \quad(j=1,2, \cdots, N),
$$

whose matrix is of rank two, may have zero, one, or two fundamental solutions. Its general solution is a linear homogeneous combination of all the fundamental solutions, the coefficients being arbitrary non-negative constants.

5. Linear homogeneous inequalities in three variables. Here it is sufficient to discuss the case where not all the representative points lie on a single plane through the origin.

A non-trivial solution is necessarily of one of the three types: (i) it contains two points of the set that are not collinear with the origin, in which case it will be called a fundamental solution; (ii) it contains one point $P$ of the set but no other point not collinear with $P$ and the origin; or (iii) it contains no point of the set.

Suppose the system proposed has a solution $\sigma$ of the second type, containing the point $P$ of the set. A plane coinciding initially with $\sigma$ can be rotated in either sense about the line $O P$ without passing through a point of the set until it contains another point of the set and thus becomes a fundamental 
solution. If the two fundamental solutions so obtained coincided, all the points would be coplanar with the origin, contrary to hypothesis. Hence there are two fundamental solutions $\sigma_{1}$ and $\sigma_{2}$, coaxial with $\sigma$, and $\sigma$ can be expressed in the form

$$
\sigma=a \sigma_{1}+b \sigma_{2},
$$

where $a$ and $b$ are constants. By considering a point of the set in $\sigma_{2}$ and not in $\sigma_{1}$ we prove that $a$ is positive. Similarly $b$ is positive.

Suppose the system proposed has a solution $\sigma$ containing no point of the set. We may rotate a plane initially coinciding with $\sigma$ about any line in $\sigma$ through the origin until the variable plane contains a point of the set. Hence there is a solution of type (i) or (ii) and consequently at least one fundamental solution. Let $\sigma_{1}$ be any fundamental solution. Rotate a plane initially coinciding with $\sigma$ about the intersection of $\sigma$ and $\sigma_{1}$ until it contains a point of the set. Let the final position be $\sigma^{\prime}$. The rotation can be accomplished in either sense. The two solutions so obtained could coincide with $\sigma_{1}$ only if the given point set were coplanar with the origin. Hence we may assume that $\sigma^{\prime}$ is distinct from $\sigma_{1}$, and we have

$$
\sigma=a \sigma_{1}+b \sigma^{\prime},
$$

where $a$ and $b$ are constants. By substitution of the coördinates of a point of the set which is on $\sigma^{\prime}$ but not on $\sigma_{1}$, we prove that $a$ is positive. Likewise $b$ is positive. Now $\sigma^{\prime}$ is a solution of type (i) or (ii). If of type (i), $\sigma^{\prime}$ is a fundamental solution in (5.2). If of type (ii), formula (5.1) applies to $\sigma^{\prime}$, and we have on substituting in (5.2)

$$
\sigma=a \sigma_{1}+b \sigma_{2}+c \sigma_{3}
$$

where $a, b$ and $c$ are positive constants.

Let $\sigma_{1}, \sigma_{2,}, \cdots, \sigma_{p}$ be the complete system of fundamental solutions, i.e., include all the fundamental solutions there are. Then by the above discussion, any solution $\sigma$ of (1.1) can be expressed in the form

$$
\sigma=\sum_{i=1}^{p} a^{i} \sigma_{i}
$$

where the $a$ 's are non-negative constants. Conversely, it is seen that (5.4) is a solution for arbitrary non-negative values of the $a$ 's because at any point of the given set the right member of (5.4) is computed by addition and multiplication from non-negative numbers.

The number of fundamental solutions in three dimensions may have any value. If we take as the given points the vertices of a convex polygon of $N^{*}$ 
sides whose plane does not contain the origin, the system has $N$ fundamental solutions, as is readily seen geometrically.

6. Rotation of an $(n-1)$-flat in $n$-dimensional space. If $\sigma$ and $\tau$ are two distinct oriented $(n-1)$-flats, they intersect in an $(n-2)$-flat which is contained by the $(n-1)$-flat

$$
\sigma \cos \alpha+\tau \sin \alpha
$$

where $\alpha$ is any constant.

For $\alpha=0,(6.1)$ is the $(n-1)$-flat $\sigma$. If $\alpha$ increases continuously from zero to any positive value $\alpha_{1}$, expression (6.1) for any value on the interval $\left(0, \alpha_{1}\right)$ represents an $(n-1)$-flat through the intersection of $\sigma$ and $\tau$. The passage from $\sigma$ to $\sigma \cos \alpha_{1}+\tau \sin \alpha_{1}$ can be defined as a rotation* in the positive sense. In the same way, if $\alpha$ decreases from zero to any negative value $\alpha_{2}$, the passage from $\sigma$ to $\sigma \cos \alpha_{2}+\tau \sin \alpha_{2}$ can be defined as a rotation in the negative sense. The intersection of $\sigma$ and $\tau$ can be called the axis of the rotation. If the coördinates of any point $P$ not on the axis are substituted in (6.1) and the result is placed equal to zero, an $\alpha$ can be found for which the $(n-1)$-flat (6.1) contains $P$. Moreover, there will be both a positive and a negative $\alpha$, i.e., the rotation can be accomplished in either one of two senses. If a finite set of points is given, we can determine the least positive $\alpha$ corresponding to each of them. The point, or points, in the set having the smallest positive $\alpha$, say $\alpha_{0}$, is the first point reached in the rotation in the positive sense. The result of substituting the coördinates of any point of the set in (6.1) is a continuous function of $\alpha$, say $f(\alpha)$, which does not vanish within the interval $\left(0, \alpha_{0}\right)$. Hence $f(\alpha)$ does not change sign on the closed interval $\left(0, \alpha_{0}\right)$, i.e., if $\sigma$ is a solution of the inequalities for the given set of points, then the final position of (6.1), namely, $\sigma \cos \alpha_{0}+\tau \sin \alpha_{0}$, is also a solution.

7. Linear homogeneous inequalities in $n$ variables. The generalization to the problem in $n$ variables follows readily from the preceding case of three variables. Here we shall discuss the case where not all the representative points lie on a single $(n-1)$-flat through the origin.

A given non-trivial solution is necessarily of one of the $n$ types (i) it contains $n-1$ points of the set that are not on an $(n-2)$-flat, in which case it will be called a fundamental solution; (ii) it contains $n-2$ points of the set which determine an $(n-2)$-flat, but no points of the set not on that $(n-2)$ flat; $\cdots ;(n)$ it contains no points of the set.

* It is not to be supposed that $\alpha$ is the measure of the angle through which the $(n-1)$-flat is rotated. The latter is a function of $\alpha$, whose explicit expression in terms of $\alpha$ can be obtained when the expression for the linear element in the coördinates $x$ is known. This expression is of no use in the present discussion. 
Suppose the system proposed has a solution $\sigma$ which contains $l$ points of the set which determine an $l$-flat but no points of the set not on that $l$-flat.

If $l<n-1$, there is an $(n-1)$-flat $\tau_{1}$ distinct from $\sigma$ and containing the $l$ points. $\sigma$ and $\tau_{1}$ intersect in an $(n-2)$-flat. Let a variable $(n-1)$-flat, initially coinciding with $\sigma$, be rotated without passing through a point of the set until it contains another point $P$ of the set and thus becomes a solution $\tau_{2}$ containing at least $l+1$ points of the set not on an $l$-flat. The rotation can be accomplished in either of two senses. If the two solutions so obtained coincided with $\tau_{1}$, all the points would be in the same $(n-1)$-flat, namely $\tau_{1}$, contrary to hypothesis. Hence we may assume $\tau_{1} \neq \tau_{2}$. Since the three $(n-1)$-flats are coaxial,

$$
\sigma=a \tau_{1}+b \tau_{2}
$$

where $a$ and $b$ are constants. At a point of $S_{n}$ in $\tau_{2}$, but not in $\tau_{1}$, the quantities $\sigma$ and $\tau_{1}$ are positive and $\tau_{2}$ is zero. Hence $a$ is positive.

If the points of $S_{n}$ in $\tau_{2}$ lie in a flat of less than $n-1$ dimensions, this process can be repeated. Finally, we obtain a solution which is the $(n-1)$-flat determined by $n-1$ points of the set, i.e., a fundamental solution containing the $l$ points which are in $\sigma$.

Let $\sigma_{1}$ be any fundamental solution containing the $l$ points. In the above argument it can replace $\tau_{1}$, so that we have

$$
\sigma=a \sigma_{1}+b \tau_{2}
$$

where $a$ is positive. It is in this case possible to prove that $b$ is also positive. $\sigma_{1}$, being distinct from $\tau_{2}$ and containing $n-1$ points of the set, contains a point of the set not in $\tau_{2}$. The substitution of this point in (7.2) makes $\sigma_{1}$ zero, and $\sigma$ and $\tau_{2}$ positive. Hence $b$ is positive.

The solution $\tau_{2}$ contains at least $l+1$ points of the set not on the same $l$-flat. The above general result shows that there is a fundamental solution $\sigma_{2}$ containing the same points of the set as $\tau_{2}$. If $\sigma_{1}$ were the same as $\sigma_{2}$, then $\sigma$ would contain at least $l+1$ points of the set not on the same $l$-flat. Hence $\sigma_{1}$ and $\sigma_{2}$ are distinct.

Moreover, we have, by the above general argument,

$$
\tau_{2}=c \sigma_{2}+d \tau_{3}
$$

where $c$ and $d$ are positive constants.

This process can be continued until a $\tau_{p}$ which contains $n-1$ points is obtained. The number of operations $p-1$ is at most equal to $n-1-l$. Hence

$$
p+l \leqq n \text {. }
$$

Since $\tau_{\nu}$ is a fundamental solution, we write it $\sigma_{p}$ and we hàve 


$$
\sigma=a^{1} \sigma_{1}+a^{2} \sigma_{2}+\cdots+a^{p} \sigma_{p},
$$

where the $a$ 's are positive constants. The $\sigma$ 's are distinct fundamental solutions. We have already seen that any two consecutive $\sigma$ 's are distinct. $\sigma_{3}$ is distinct from $\sigma_{1}$ because it has a point in common with $\sigma_{2}$ which is not contained in $\sigma_{1}$. Similarly for any other non-consecutive pair.

The totality of fundamental solutions containing the $l$ points is called the complete system of fundamental solutions containing those points. The number of such solutions is necessarily finite because only a finite number of $(n-1)$ flats are determined by a finite number of points.

If there is a fundamental solution $\sigma_{p+1}$ containing the $l$ points and distinct from $\sigma_{1}, \cdots, \sigma_{p}$, we may repeat the above process, getting

$$
\sigma=a^{p+1} \sigma_{p+1}+\cdots+a^{q} \sigma_{q},
$$

where the $a$ 's are positive constants, and $\sigma_{p+1}, \cdots, \sigma_{q}$ are distinct fundamental solutions containing the $l$ points. We do not know they are all distinct from $\sigma_{1}, \cdots, \sigma_{p}$. The addition of (7.4) and (7.5) gives an expression for $\sigma$ in which $\sigma_{1}, \cdots, \sigma_{p+1}$ surely occur with positive coefficients. If there is a fundamental solution through the $l$ points not appearing in the right hand member, we may repeat the process.

If a solution $\sigma$ contains $l$ points of the set and in addition other points of the set not on the $l$-flat determined by them, there is a set of $l^{\prime}$ points containing the $l$ points, to which the above argument is applicable. Hence any solution through $l$ points of the set can be expressed as a linear homogeneous combination of the complete system of fundamental solutions through those $l$ points, the coefficients being non-negative. Conversely, any linear homogeneous combination of the complete system of fundamental solutions through the $l$ points, the coefficients being arbitrary non-negative constants, obviously passes through the $l$ points, and is a solution because its value at $S_{n}$ is computed by multiplication and addition from non-negative numbers.

THEOREM 4. If the representative points do not all lie in an $(n-1)$-flat through the origin, the general solution of

$$
\sum_{i=1}^{n} \lambda^{i} x_{i}^{j} \geqq 0 \quad(j=1,2, \cdots, N),
$$

containing a specified sub-set of the given set of points $S_{n}$, is a linear homogeneous combination of the complete system of fundamental solutions containing those points, the coefficients being arbitrary non-negative constants.

A corollary is Minkowski's theorem: 
THEOREM 5. The general solution of the system

$$
\sum_{i=1}^{n} \lambda^{i} x_{i}^{i} \geqq 0 \quad(j=1,2, \cdots, N),
$$

whose representative points do not all lie in an $(n-1)$-flat through the origin, is a linear homogeneous combination of the complete system of fundamental solutions, the coefficients being arbitrary non-negative constants.

If the complete system mentioned in either of the two foregoing theorems is vacuous, the general solution is the trivial solution.

Suppose a solution $\sigma$ contains a set $A$ of points and contains no point of another set $B$. By Theorem 4 , we know that $\sigma$ can be expressed in the form (7.4), where the $a$ 's are non-negative and the $\sigma$ 's are fundamental solutions through $A$. In order that $\sigma$ do not contain a given point of $B$, at least one $\sigma_{i}$ must not contain that point. Hence, a necessary condition for the existence of $\sigma$ is that corresponding to every point of $B$ there be at least one fundamental solution through the points $A$ not containing it.

Any fundamental solution through $A$ which does not contain all the points $B$ can be employed as the $\sigma_{1}$ in (7.2). If we use every such fundamental solution in turn as the $\sigma_{1}$ in (7.2) and add the resulting (7.4)'s, we have $\sigma$ expressed as a linear homogeneous expression in which every fundamental solution passing through $A$ and not containing all points of $B$ occurs with a positive coefficient, and we are sure that the other coefficients are non-negative.

Conversely, if the necessary condition of the theorem is fulfilled, a linear homogeneous combination of the complete system of fundamental solutions through $A$ in which every fundamental solution not containing all points of $B$ has an arbitrary positive coefficient and those containing all points of $B$ have non-negative coefficients satisfies the system, obviously contains $A$, and contains at least one non-vanishing $\sigma$ corresponding to every point of $\dot{B}$. Hence we have the

Fundamental Theorem 6. The system

$$
\sum_{i=1}^{n} \lambda^{i} x_{i}^{j} \geqq 0,
$$

whose representative points do not all lie in an (n-1)-flat through the origin, has a solution containing every point of a specified sub-set $A$ of the given set of points and containing no point of another specified sub-set $B$ if and only if corresponding to every point of $B$ there is at least one fundamental solution through $A$ not containing it. When a solution exists, the general solution is a linear homogeneous combination of the complete system of fundamental solutions through $A$, the 
coefficient of every fundamental solution not containing all points $B$ being an arbitrary positive constant, and that of every fundamental solution containing all points $B$ being an arbitrary non-negative constant.

Making the point set $B$ contain all points of $S_{n}$ not in the flat space determined by $A$, since no fundamental solution through $A$ contains all points of $B$ in this case, we get

THEOREM 7. If the representative points do not all lie in an (n-1)-fat through the origin, the general solution of the system

$$
\sum_{i=1}^{n} \lambda^{i} x_{i}^{j} \geqq 0
$$

which contains a subset $A$ of the given set and contains no points of the given set not on the flat space determined by $A$, is a linear homogeneous combination of the complete system of fundamental solutions through $A$, the coefficients being arbitrary positive constants.

If the complete system of fundamental solutions referred to in the preceding theorem is vacuous, there is no solution to the problem.

If $A$ contains no points, we have a new solution of the problem (1.2) considered by Dines:

THEOREм 8. If the representative points do not all lie in an (n-1)-flat through the origin, the general solution of the system

$$
\sum_{i=1}^{n} \lambda^{i} x_{i}^{j} \geqq 0
$$

which contains no points of the given set is a linear homogeneous combination of the complete system of fundamental solutions, the coefficients being arbitrary positive constants.

Again, if the complete system referred to is vacuous, there is no solution.

8. Necessary and sufficient conditions for the existence of a non-trivial solution. In the preceding section we proved that a system of inequalities (1.1) has a non-trivial solution only if it has a fundamental solution. This condition is obviously also sufficient. We propose in the present section to get the necessary and sufficient condition in a slightly different form.

Given a set of points $S_{n}$ having a fundamental solution $\Pi_{n-1}$ containing $l$ points of the set which determine an $l$-flat. Consider the points of $S_{n}$ contained in $\Pi_{n-1}$. They form a set which we call $S_{n-1}$. Let $\Pi_{n-2}$ be a fundamental solution, through the same $l$ points, for the set $S_{n-1}$. And so on until a $\mathrm{II}_{m}$ is reached, which is a fundamental solution for the points $S_{m+1}$ of $S_{n}$ in 
$\Pi_{m+1}$, and is such that the points $S_{m}$ in $\Pi_{m}$ have only the trivial solution. $S_{m}$ will be called the inconsistent set containing the given $l$ points. Clearly $l \leqq m \leqq n$.

THEOREM 9. Every solution for the set $S_{n}$ which passes through $l$ given points must contain the corresponding inconsistent set $S_{m}$.

Any solution $\sigma$ for the set $S_{n}$ which is distinct from $\mathrm{II}_{n-1}$ will, by Theorem I, meet $\Pi_{n-1}$ in a solution for $S_{n-1}$. The latter solution will meet $\Pi_{n-2}$ in a solution for $S_{n-2}$, and so on until we reach a solution for set $S_{m+1}$. If that solution were distinct from $\Pi_{m}$, it would meet $\Pi_{m}$ in a non-trivial solution for $S_{m}$. As such a solution does not exist, the solution for the set $S_{m+1}$ finally reached must coincide with $\Pi_{m}$. Hence $\sigma$ contains $\Pi_{m}$.

THEOREM 10. A solution containing a given set of $l$ points and the l-fat determined by them, but no other points of the set, exists, if and only if $l=m$, that is, if the inconsistent set for the $l$ points is contained by the l-flat determined by them.

THEOREM 11. There are exactly $n-m$ linearly independent fundamental solutions through $l$ points determining an l-flat, where $m$ is the dimensionality of the inconsistent set containing the $l$ points.

In the space $\Pi_{m+1}$ above there is one and only one fundamental solution $\Pi_{m}$ for the set $S_{m+1}$. Let us assume that in $\Pi_{m+a}$ there are $a$ linearly independent fundamental solutions for $S_{m+a}$. In $\Pi_{m+a+1}$ we know the existence of one fundamental solution, namely, $\Pi_{m+a}$. We can rotate an $(m+a)$-flat, initially coinciding with $\Pi_{m+a}$, about any one of the fundamental solutions in $\Pi_{m+a}$ for $S_{m+a}$. This rotation can be carried out in either of two senses. Since by the result of $\$ 3$ any $(m+a)$-flat which passes through a fundamental solution for $S_{m+a}$ and which is contained by $\Pi_{m+a+1}$ is a solution in $\Pi_{m+a+1}$ for $S_{m+a}$, when properly oriented, we know that the points of $\Pi_{m+a}$ which do not remain in the variable $(m+a)$-flat are all on the same side of that $(m+a)$-flat. Hence for one sense of rotation the variable $(m+a)$-flat will remain a solution for $S_{m+a+1}$, and as soon as it contains a point of $S_{m+a+1}$ not on $\Pi_{m+a}$, will be fundamental for $S_{m+a+1}$. In this way we deduce $a$ fundamental solutions for $S_{m+a+1}$, which together with $\Pi_{m+a}$ constitute a set of $a+1$ fundamental solutions for $S_{m+a+1}$.

If these solutions were linearly dependent, they would have in common a point $P$ not on $\Pi_{m}$. Since one of these solutions, namely $\Pi_{m+a}$, meets the others in fundamental solutions for the set $S_{m+a}$, this point $P$ would be on the $a$ fundamental solutions for $S_{m+a}$. But this contradicts their linear independence. Hence by induction we conclude that the set $S_{m+a}$ proposed for solution in the space $\Pi_{m+a}$ has $a$ linearly independent solutions passing through 
the $l$ given points for all possible values of $a$. The theorem stated follows by making $a=n-m$.

9. Solution of numerical problems. In the preceding sections we have developed the theory which will enable us to solve any of the problems mentioned in the introduction. The actual solution requires the knowledge of the complete system of fundamental solutions of the system (1.1) corresponding to all the points representing the given conditions; for example, if the system proposed is

$$
u_{1}>0, u_{2}=0, u_{3} \geqq 0,
$$

the system of which it is necessary to know the complete system of fundamental solutions is

$$
u_{1} \geqq 0, \quad u_{2} \geqq 0, \quad u_{3} \geqq 0 .
$$

If the general solution of only the problem (1.1) is desired, the simplest way to compute the fundamental solutions is as follows. Assuming unknowns have been omitted, if necessary, so that the rank of the system is equal to the number of remaining unknowns, write the equation of every $(n-1)$-flat determined by $n-1$ points of the set. Substitute successively in each the coordinates of all the points of the set. If for a given $(n-1)$-flat the substitution gives a variation in sign, the $(n-1)$-flat is not a fundamental solution. If the substitution gives only non-negative values, the left member of the equation of the $(n-1)$-flat defines an oriented $(n-1)$-flat which is a fundamental solution. If the substitution gives only non-positive values, the left member of the equation with its sign changed gives a fundamental solution.

If, however, the solution of one of the associated problems is desired, it is convenient to make use of a rectangular array whose formation we proceed to describe. Each column is headed by a combination of the indices of the points of the set taken $n-1$ at a time, each combination being written once and only once in a definite but arbitrary order. The rows are numbered with the indices of the points of the set. The entry made in any position is the sign of the determinant having as its rows the coördinates of the points involved, written in the order determined by the indices at the head of the column followed by the index indicating the row. A zero is used to denote absence of sign. Any column containing no variation in sign is headed by a combination of points which determine a fundamental solution, if they do not lie in an $(n-2)$-flat. A zero in the array indicates that the point corresponding to the row is in the $(n-1)$-flat determined by the indices at the top of the column. 
The totality of fundamental solutions containing a given set of points is readily picked out by means of this array, and it is easy to tell whether any point of the set is in a given fundamental solution. It is also possible from the disposition of zeros in the columns involved to tell whether two fundamental solutions are distinct.

An examination of the array will show what points are common to all fundamental solutions through a given set of $l$ points, that is, will determine the set $S_{m}(\$ 8)$ for those $l$ points.

10. Skeleton sets. If in the sign matrix of the last section every column which is headed by a combination containing a given index has a variation in sign, the point corresponding to that index is in no fundamental solution. As the general solution of (1.1) involves only the fundamental solutions, any point in no fundamental solution can be omitted without altering the solution of (1.1), provided its omission leaves the set $n$-dimensional. The corresponding condition (1.1) is satisfied as a consequence of the others, and its left member is positive for all solutions.

By a skeleton set of (1.1) we mean a minimum set of the given points which are necessary to determine the solution. This set is determined in the following manner.

From the $n$-dimensional set $S_{n}$ form the array described in the preceding section. Omit as many points. which are in no fundamental solution as will leave the dimensionality $n$, using the test just given. Consider all the points of $S_{n}$ which are in a particular fundamental solution $\Pi_{n-1}$. Call them $S_{n-1}$. Form a new array with the points of $S_{n-1}$. If $S_{n-1}$ is consistent, it will have at least one fundamental solution. Consider a point $P$ of $S_{n-1}$ contained in no fundamental solution in $\Pi_{n-1} . P$ is contained by no solution of the problem in $\Pi_{n-1}$ defined by $S_{n-1}$. Any solution of the problem in $n$ dimensions, other than $\Pi_{n-1}$, will intersect $\Pi_{n-1}$ in a solution for $S_{n-1}$ (Theorem 1). Hence $P$ is on no solution of the original $n$-dimensional problem except $\Pi_{n-1}$, and if $S_{n-1}$ remains $(n-1)$-dimensional upon its omission, $P$ can be omitted from $S_{n}$. The condition (1.1) corresponding to $P$ will be a consequence of the remaining conditions (1.1), and its left member will be positive for every solution of (1.1) other than $\Pi_{n-1}$, which is the only fundamental solution through $P$. Let $\Pi_{n-2}$ be a fundamental solution for $S_{n-1}$, call the points of the set in it $S_{n-2}$, and repeat the above operation until finally a $k$-dimensional set $S_{k}$ is reached which is inconsistent in $\mathrm{II}_{k}$, i.e., has no funclamental solution if proposed as a problem in $k$ dimensions.

The sign matrix of $S_{k}$, as a set in $k$ dimensions, has a variation in sign in every column which contains a non-zero element. There must be at least one such column because the set is $k$-dimensional. Any puint whose omission 
gives an array with at least one non-zero column and a variation in sign in every such column can be omitted from the set because the resulting set will be both $k$-dimensional and inconsistent.

Any column contains $k-1$ zeros. In order that a column contain a variation in sign, the number of points in a $k$-dimensional inconsistent set must be at least $k+1$. It is not always possible, conversely, to reduce an inconsistent set of $k$ dimensions to a skeleton set of $k+1$ points: for example, the inconsistent set composed of the vertices of a square whose center is the origin cannot be reduced to $k+1=3$ points because the omission of any one of the points leaves a consistent set having the diagonal connecting the two adjacent vertices as a fundamental solution.

When all possible points have been omitted by considering all fundamental solutions and all fundamental solutions contained in them, the remnant of the set is a skeleton set.

A particular consequence of the above discussion is

THEOREM 12. Any set of $n$ or fewer inequalities (1.1) in $n$ unknowns has a fundamental solution.

To illustrate the formation of the skeleton set, consider the case of three dimensions. Draw the planes through the origin determined by every pair of points of the set. Omit any plane which separates two points of the set. The planes remaining after the omission will be fundamental solutions. The only points retained are those in fundamental solutions, with the exception that when there is only one fundamental solution, a single point not on it is retained in order to leave the set three-dimensional.

Next consider in a fundamental solution the lines joining the points of the set to the origin, and omit any one of them which separates two points of the set, together with all points of the set on it. Repeat for the other fundamental solutions, if any exist.

Next consider one of the lines which have not been omitted. If the origin separates two points of the set on it, we retain those points and omit the others. If the points on it are all on the same side of the origin, we retain any one of them and omit the others.

If the original set is inconsistent, i.e., has no fundamental solution, we examine each point in turn to see if it can be omitted without making the remaining set consistent. By trial, we thus find a three-dimensional inconsistent set, the omission of any one of whose points leaves a consistent set.

If the set of points in a fundamental solution is inconsistent, the process just outlined for inconsistent three-dimensional sets is to be used to reduce it.

The points of the original set which remain after the above omissions form a skeleton set for the given three-dimensional set. 
If (1.1) has a solution not containing any point of the set, the geometric configuration defined by the skeleton set is a convex pyramidal space, having as its vertex the origin, and as faces all the planes giving fundamental solutions. The consecutive planes intersect along the lines joining the points of the skeleton set to the origin.

Minkowski has also given a criterion for finding when one of the inequalities is a consequence of the others.*

11. Examples. Solve the system:

$$
\begin{aligned}
& \lambda-\mu+\nu \geqq 0, \quad \nu \geqq 0, \quad \mu \geqq 0, \\
& 2 \lambda \geqq 0, \quad \lambda+\mu \geqq 0, \lambda+\mu+\nu \geqq 0 .
\end{aligned}
$$

The set of points defined by the system is

$$
(1,-1,1), \quad(0,0,1), \quad(0,1,0), \quad(2,0,0), \quad(1,1,0), \quad(1,1,1) .
$$

We plot these points. The figure shows that there are four fundamental solutions. The same result is obtained from the rectangular array:

$$
\begin{array}{cccccccccccccccc} 
& 12 & 13 & 14 & 15 & 16 & 23 & 24 & 25 & 26 & 34 & 35 & 36 & 45 & 46 & 56 \\
1 & 0 & 0 & 0 & 0 & 0 & - & - & - & - & - & - & 0 & + & + & + \\
2 & 0 & + & + & + & + & 0 & 0 & 0 & 0 & - & - & - & + & + & 0 \\
3 & - & 0 & + & + & 0 & 0 & + & + & + & 0 & 0 & 0 & 0 & - & - \\
4 & - & - & 0 & - & - & - & 0 & - & - & 0 & 0 & + & 0 & 0 & + \\
5 & - & - & + & 0 & - & - & + & 0 & 0 & 0 & 0 & + & 0 & - & 0 \\
6 & - & 0 & + & + & 0 & - & + & 0 & 0 & - & - & 0 & + & 0 & 0
\end{array}
$$

The complete set of fundamental solutions is $x ; z ; y+z$; and $x+y$.

The general solution is

$$
a x+b z+c(y+z)+d(x+y),
$$

where $a, b, c, d$ are arbitrary non-negative constants.

A particular solution containing no points of the set is

$$
\sigma=2 x+y+3 z \text {. }
$$

Let us express this in terms of the fundamental solutions by the method of $\$ 7$.

The family of planes passing through the intersection of $\sigma$ and the fundamental solution $\sigma_{1}=x$ is

$$
(2 x+y+3 z)+a x
$$

\footnotetext{
* Loc. cit., pp. 43-45.
} 
The $a$ 's corresponding to the other points of the set are $-4,-2,-3$, -6 . We choose the $a$ numerically smaller than any of the other $a$ 's and substitute in (11.1), whence

$$
\tau_{2}=y+3 z
$$

The family of planes through the intersection of $\tau_{2}$ and the fundamental solution $\sigma_{2}=z$ is

$$
y+3 z+b z .
$$

The $b$ 's corresponding to all the other points of the set are $-2,-3,-3,-4$. Hence

$$
\sigma_{3}=y+z \text {. }
$$

Since $\sigma_{3}$ contains two points of the set, it is a fundamental solution, and

$$
\sigma=2 x+2 z+(y+z) .
$$

Starting anew with the fundamental solution $\sigma_{1}=x+y$, we get

$$
\sigma=(x+y)+3 z+x .
$$

The addition of the two expressions for $\sigma$ gives

$$
2 \sigma=3 x+5 z+(y+z)+(x+y) .
$$

This is a linear homogeneous combination of the complete system of fundamental solutions, the coefficients being positive constants.

Example 2. Suppose the system proposed for solution is

$$
(0,0,1),(-1,0,0),(0,1,0),(1,0,0) \text {. }
$$

There are just two fundamental solutions and they both contain $(-1$, $0,0),(1,0,0)$, which therefore constitute the inconsistent set. The general solution for the case $\geqq 0$ is $a y+b z$, where $a$ and $b$ are arbitrary non-negative constants.

Example 3. The points $(1,-1,-1),(2,0,0),(2,2,2)$ lie in the plane $y-z=0$. In that plane $x$ and $y$ can be taken as coördinates. The solution of system (1.1) corresponding to the given points $(1,-1),(2,0),(2,2)$ is readily found to be $(a+2 b) x+(a-2 b) y$. The general solution of the problem in three unknowns is given by the pencil of planes through this line and is obtained by adding $c(y-z)$ to the above. Thus the general solution is furnished by

and is

$$
(a+2 b) x+(a-2 b+c) y-c z,
$$

$$
\lambda=a+2 b, \mu=a-2 b+c, \nu=-c,
$$

where $a$ and $b$ are arbitrary non-negative constants and $c$ is an arbitrary constant. 
12. An application. The problem considered by Lovitt* is to find the conditions of compatibility of the system

$$
\begin{gathered}
\lambda-\mu>0, \mu-\nu>0, \\
\left(A_{1}-B_{1}\right) \lambda+\left(A_{2}-B_{2}\right) \mu+\left(A_{3}-B_{3}\right) \nu>0, \\
\left(A_{1}-C_{1}\right) \lambda+\left(A_{2}-C_{2}\right) \mu+\left(A_{3}-C_{3}\right) \nu>0,
\end{gathered}
$$

where the $A, B, C$ are given non-negative numbers satisfying the relations

$$
\sum A=\sum B=\sum C \text {. }
$$

The matrix of the coefficients is

$$
\left\|\begin{array}{ccc}
1 & -1 & 0 \\
0 & 1 & -1 \\
A_{1}-B_{1} & A_{2}-B_{2} & A_{3}-B_{3} \\
A_{1}-C_{1} & A_{2}-C_{2} & A_{3}-C_{3}
\end{array}\right\|
$$

The sum of the elements on each row being zero by virtue of (12.2), the representative points all lie in the plane

$$
x+y+z=0 \text {. }
$$

The convex polygon defined by a set of coplanar points is the figure assumed by a stretched rubber band placed around pins fixed at the points. It can be obtained geometrically by drawing all segments determined by two points of the set, and erasing all those segments which, produced if necessary, separate two points of the set. It reduces to a straight line if the given points are collinear.

Hence a geometric form of the necessary and sufficient condition that (12.1) have a solution is as follows: the origin must not be inside the convex polygon defined by the representative points nor on its boundary. Since the property involved in this condition is unaltered by orthogonal projection on another plane through the origin, in applying the test for consistency the representative points can be replaced by their projections on one of the coordinate planes.

To obtain the condition in analytic form, we may take $x, y$ as the coordinates in the plane $x+y+z=0$. The sign matrix is then

$\begin{array}{ccccc} & 1 & 2 & 3 & 4 \\ 1 & 0 & - & A_{3}-B_{3} & A_{3}-C_{3} \\ 2 & + & 0 & A_{1}-B_{1} & A_{1}-C_{1} \\ 3 & B_{3}-A_{3} & B_{1}-A_{1} & 0 & P \\ 4 & C_{3}-A_{3} & C_{1}-A_{1} & -P & 0\end{array}$,

${ }^{*}$ Loc. cit. 
where

$$
P=A_{2}\left(B_{1}-C_{1}\right)+B_{2}\left(C_{1}-A_{1}\right)+C_{2}\left(A_{1}-B_{1}\right) .
$$

We desire the condition that the associated system with symbols $\geqq 0$ have a solution containing no point of the set. The fundamental theorem (\$7) gives immediately the result, which can be stated as follows: if any column, say the ith, is deleted from the matrix, there must remain a column containing no variation of sign and containing a non-zero element on the ith row.

It is easy to deduce from the above the two italicized results given by Lovitt on page 365 of his article. The first situation is described by (we have interchanged the significance of Lovitt's $A$ and $B$ )

$$
A_{1}=B_{1}, A_{2}<B_{2}, A_{3}>B_{3}, B_{i}=C_{i},
$$

and the matrix is

$$
\begin{array}{rrrr}
0 & - & + & + \\
+ & 0 & 1 & 0 \\
- & 0 & 0 & 0 \\
- & 0 & 0 & 0 .
\end{array}
$$

There is no solution because after the deletion of the fourth column the only column with a non-zero element on the fourth row contains a variation of sign.

The second situation is

$$
A_{1}<B_{1}, A_{2}=B_{2}, A_{3}>B_{3},
$$

for which the matrix is

$$
\begin{array}{cccc}
0 & - & + & A_{3}-C_{3} \\
+ & 0 & - & A_{1}-C_{1} \\
- & + & 0 & P \\
C_{3}-A_{3} & C_{1}-A_{1} & -P & 0
\end{array}
$$

When the last column is omitted, the remaining columns all have variations of sign. Hence there is no solution.

Strictly speaking, the system treated by Lovitt contains another condition,

$$
\nu>0
$$

which insures that $\lambda, \mu, \nu$ are positive. For the sake of simplicity we have omitted this condition. It is easy to see, however, that the inclusion of the additional point $(0,0,1)$ will not alter the result given above. For any plane passed through a line giving a solution in the plane $x+y+z=0$ obviously can 
be rotated until all the points, including $(0,0,1)$, are on its positive side; and, conversely, the trace of any solution on the plane $x+y+z=0$ is a solution in that plane by Theorem 1 .

13. Non-homogeneous case. With the system of non-homogeneous inequalities

$$
x_{0}^{i}+\sum_{i=1}^{n} \lambda^{i} x_{i}^{j} \sim 0 \quad(j=1, \cdots, N),
$$

there is associated a homogeneous system

$$
\begin{gathered}
\lambda^{0} x_{0}^{j}+\sum_{i=1}^{n} \lambda^{i} x_{i}^{j} \sim 0, \\
\lambda^{0}>0,
\end{gathered}
$$

where the symbol $\sim$ represents any one of the signs $\geqq,>,=$.

If a set of $\lambda^{\prime}$ 's satisfy (13.1), then $1, \lambda^{1}, \lambda^{2}, \cdots, \lambda^{n}$ satisfy (13.2).

Conversely, if a solution of (13.2) is $\lambda^{0}, \lambda^{1}, \lambda^{2}, \cdots, \lambda^{n}$, then

$$
x_{0}^{j}+\sum_{i=1}^{n}\left(\lambda^{i} / \lambda^{0}\right) x_{i}^{j} \sim 0,
$$

that is, $\lambda^{1} / \lambda^{0}, \lambda^{2} / \lambda^{0}, \cdots, \lambda^{n} / \lambda^{0}$ is a solution of (13.1). Hence the solution of (13.1) can be determined from that of (13.2).

The methods of the preceding sections may, therefore, be applied to give solutions of systems of non-homogeneous inequalities of the general type.

The above reduction is employed both by Minkowski and Dines. The adjunction of $\lambda^{0}>0$ to Dines' system gives a system of the same sort, but its adjunction to Minkowski's system gives one of the intermediate types considered in the present paper. As Minkowski gives the general solution only of (1.1), his treatment of the non-homogeneous case is incomplete.

14. Positive solutions of systems of homogeneous or non-homogeneous linear equations. It suffices to discuss the case of homogeneous equations, because it is always possible to convert a non-homogeneous into a homogeneous system by the use of homogeneous coördinates, as in $\$ 13$.

This case is covered completely by the fundamental theorem of $\$ 7$. The method of solving is given by the following

Example. Find the positive solutions of the equation

$$
\lambda^{1}+2 \lambda^{2}-\lambda^{3}-\lambda^{4}=0 .
$$

To solve, we must find the fundamental solutions of

$$
\lambda^{1}+2 \lambda^{2}-\lambda^{3}-\lambda^{4} \geqq 0, \lambda^{1} \geqq 0, \lambda^{2} \geqq 0, \lambda^{3} \geqq 0, \lambda^{4} \geqq 0 .
$$


For the set of points

$$
(1,2,-1,-1),(1,0,0,0),(0,1,0,0),(0,0,1,0),(0,0,0,1)
$$

we form the array

$\begin{array}{ccccccccccc} & 123 & 124 & 125 & 134 & 135 & 145 & 234 & 235 & 245 & 345 \\ 1 & 0 & 0 & 0 & 0 & 0 & 0 & - & + & + & - \\ 2 & 0 & 0 & 0 & + & - & - & 0 & 0 & 0 & - \\ 3 & 0 & - & + & 0 & 0 & + & 0 & 0 & + & 0 \\ 4 & + & 0 & + & 0 & - & 0 & 0 & - & 0 & 0 \\ 5 & - & - & 0 & + & 0 & 0 & + & 0 & 0 & 0 .\end{array}$

The complete set of fundamental solutions containing the first point is

$$
y+2 t ; y+2 z ; x+t ; x+z .
$$

Hence the general solution is

$$
a(y+2 t)+b(y+2 z)+c(x+t)+d(x+z),
$$

where $a, b, c$ and $d$ are arbitrary positive constants. Thus

$$
\lambda^{1}=c+d, \lambda^{2}=a+b, \lambda^{3}=2 b+d, \lambda^{4}=2 a+c .
$$

15. Another form of the necessary and sufficient condition for a solution. The geometric condition given in $\$ 12$ can be extended, as we proceed to show. First we must generalize the notion of the convex polygon defined by a set of points.

We shall not suppose, as previously, that all the flats contain the origin.

Consider a set of points $S$ which are in a $k$-flat $F_{k}(S)(0 \leqq k \leqq n)$, but are not all in a $(k-1)$-flat. Imagine constructed the set of all $(k-1)$-flats determined by $S$. Omit from it any $(k-1)$-flat which separates two points of $S$, and denote the remaining $(k-1)$-flats by $F_{k-1}(S)$.

Consider next the set of all points of $S$ in a member of $F_{k-1}$. Construct the $(k-2)$-flats and discard as before. Denote by $F_{k-2}(S)$ the totality of $(k-2)$ flats remaining after this process has been applied to all the members of $F_{k-1}$.

If this process is continued, we finally obtain a set of lines $F_{1}(S)$, none of which separates any two points of $S$ coplanar with it. Each of these lines, being determined by points of $S$, contains at least two distinct points of $S$. The application of the above general process to each line leaves two points which are separated by every other point of $S$ on their join. The points of $S$ finally remaining are denoted by $F_{0}(S)$ and are said to determine the convex figure associated with $S$. The points in $F_{0}(S)$ are called its vertices. 
From the method of the construction no $(k-1)$-flat of $F_{k-1}(S)$ separates two points of $S$. Consequently, as in $\S 7$, any $(k-1)$-flat of $F_{k-1}$ can be rotated about the $(k-2)$-flat determined by $k-1$ points of $F_{0}(S)$ in it in such a sense that the points of $S$ continue to be not separated by it. When it acquires another point of $S$, it again becomes a member of $F_{k-1}(S)$. If the initial and final positions coincided, all the points of $S$ would lie in a $(k-1)$-flat, contrary to hypothesis. Hence $F_{k-1}(S)$ contains at least two $(k-1)$-flats, i.e., the set $F_{0}(S)$ is not contained by a single $(k-1)$-flat. Therefore we have

THEOREM 13. The convex figure associated with a set of points has the same dimensionality as the set of points.

By an induction entirely analogous to that used in proving Theorem 11, we prove

THEOREM 14. There are $p$ linearly independent $(p-1)$-fats in $F_{p-1}(S)$ which contain a given vertex of $F_{0}(S)$.

A particular consequence is obtained by making $p=k$ :

THEOREM 15. There are $k$ linearly independent $(k-1)$-flats of $F_{k-1}(S)$ through every verlex of $F_{0}(S)$.

By an equation $F_{0}(S)=F_{0}(T)$ we mean that the two point sets involved are identical.

A point $P$ of space for which

$$
F_{0}(S+P) \neq F_{0}(S)
$$

is said to be exterior to the convex figure $F_{0}(S) . P$ is then necessarily a vertex of $F_{0}(S+P)$.

Relation (15.1) surely holds if $P$ is not on the $k$-flat containing $S$.

Any point $P$ which is on a $(k-1)$-flat of $F_{k-1}(S)$ and for which

$$
F_{0}(S+P)=F_{0}(S)
$$

holds is said to be on the boundary of the convex figure $F_{0}(S)$. The totality of points $P$ in any $(k-1)$-flat of $F_{k-1}(S)$ and on the boundary of $F_{0}(S)$ constitute a face of the convex figure. By Theorem 15 there are at least $k$ faces through each vertex. The regular octahedron furnishes an example of a case where there are more than this minimum.

A point $P$ which is on no $(k-1)$-flat of $F_{k-1}(S)$ and for which (15.2) holds is said to be interior to the convex figure $F_{0}(S)$.

We are now in a position to prove

THEOREM 16. The system

$$
\sum_{i=1}^{n} \lambda^{i} x_{i}^{j}>0
$$


has a solution if and only if the origin is exterior to the convex figure associated with the representative points.

The condition is necessary. Suppose the origin $P$ is not exterior. Because of equation (15.2), either $P$ is a vertex of $F_{0}(S)$ or it is collinear with two points of $S$ which it separates. In the former case, every solution of the associated system (1.1) contains a point of the set $S$, namely, $P$. In the latter case, any flat through the origin contains the two points of the set or separates them. In either case, therefore, there is no solution.

The condition is sufficient. When it is fulfilled, the origin is a vertex of $F_{0}(S+P)$. Hence by Theorem 15 there are $n$ linearly independent faces of $F_{0}(S)$ through $P$, i.e., $n$ linearly independent fundamental solutions for the set $S$, where $n$ is the dimensionality of the point set $S+P$ (either $k$ or $k+1$ ). From Theorem 11 the dimensionality of the inconsistent set for a set of $l=0$ points is zero. Hence by Theorem 10 there is a solution.

In the same way we readily prove

THEOREM 17. The system

$$
\sum_{i=1}^{n} \lambda^{i} x_{i}^{j} \geqq 0
$$

has a solution other than an equality solution if and only if the origin is not interior to the convex figure associated with the representative points.

It is to be noted that the last two theorems are true whatever the rank of the matrix of the coefficients may be.

The writer wishes to acknowledge indebtedness to Professor J. M. Thomas under whose direction this work was done.

DUKE UNIVERSITY, Durham, N. C. 